\title{
Autoregulation and Dual Stepping Mode of MYA2, an Arabidopsis Myosin XI Responsible for Cytoplasmic Streaming
}

\section{Takashi Haraguchi}

Chiba University

Kohji Ito

Chiba University

Takamitsu Morikawa

RIKEN Center for Biosystems Dynamics Research, RIKEN

Nao Shoji

Chiba University

Mitsuhiro Iwaki

RIKEN Center for Biosystems Dynamics Research, RIKEN

Motoki Tominaga ( $\nabla$ motominaga@waseda.jp )

Waseda University

\section{Research Article}

Keywords: Arabidopsis thaliana, Autoregulation , dual stepping mode of MYA2, cytoplasmic streaming

Posted Date: September 2nd, 2021

DOl: https://doi.org/10.21203/rs.3.rs-857859/v1

License: (1) This work is licensed under a Creative Commons Attribution 4.0 International License.

Read Full License

Version of Record: A version of this preprint was published at Scientific Reports on February 24th, 2022.

See the published version at https://doi.org/10.1038/s41598-022-07047-0. 


\section{Abstract}

Arabidopsis thaliana has 13 genes belonging to the myosin XI family. Myosin XI-2 (MYA2) plays a major role in the generation of cytoplasmic streaming in cells. In this study, we investigated the molecular properties of MYA2 expressed by the baculovirus transfer system. Actin-activated ATPase activity and in vitro motility assays revealed that activity of MYA2 was regulated by the globular tail domain (GTD), When the GTD is not bound to the cargo, the GTD inhibits ADP dissociation from the motor domain. Optical nanometry of single MYA2 molecules, combining TIRF microscopy and the FIONA method, revealed that the MYA2 processively moved on actin with three different step sizes: $-28 \mathrm{~nm}, 29 \mathrm{~nm}$, and $60 \mathrm{~nm}$, at low ATP concentrations. This result indicates that MYA2 uses two different stepping modes, hand-over-hand and inchworm-like. Force measurement using optical trapping showed the stall force of MYA2 was $0.85 \mathrm{pN}$, which was less than half that of myosin $\mathrm{V}(2-3 \mathrm{pN})$. These results indicated that MYA2 is more flexible than the myosin $\mathrm{V}$ responsible for vesicle transport in animal cells. Such flexibility may enable multiple myosin XIs to transport organelles quickly and smoothly, for the generation of cytoplasmic streaming in plant cells.

\section{Introduction}

Myosin is a motor protein that converts the chemical energy liberated by ATP hydrolysis into directed movement along actin filaments. Phylogenetic analyses of myosin sequences have shown that there are at least 79 myosin classes in eukaryotes ${ }^{1}$. The Myosin superfamily shares a common domain composition: a conserved motor domain (MD) with ATPase and actin-binding activities, a neck domain comprising one to six repeats of isoleucine-glutamine (IQ) motifs acting as lever arm, a coiled-coil domain, and a globular tail domain (GTD) that binds the cargo ${ }^{2}$. Many myosins of different classes participate in transport of various cargos, such as organelles, vesicles, and ribonucleoproteins, within cells $^{3}$. Myosins bind different cargos via the GTD and transport them along actin filaments by the MD activity. Proper delivery and regulation of intracellular transport by myosins within cells is crucial for maintaining cellular function. The myosin motility is finely regulated by various mechanisms such as phosphorylation, calcium ions and autoinhibition ${ }^{4}$. It has been reported that activity of myosin $\mathrm{V}$, myosin VIIA, and myosin X is regulated by autoinhibition using the GTD. In these myosins, the MD is typically maintained in an OFF state when the GTD is folded over and interacts with the motor to inhibit ATPase activity. Regulation by autoinhibition could avoid unproductive interactions with actin filaments, and is essential for the recycling of free myosins after unloading cargo. Conserved amino acid residues on both the MD and the GTD of myosin V mediate this interaction ${ }^{6-8}$.

Cytoplasmic streaming is the long-range, rotational movement of cytoplasm, widely observed in cells of organisms ranging from algae to angiosperms. Cytoplasmic streaming is generated by organelleassociated plant specific class XI myosin sliding along actin filaments ${ }^{9}$. Myosin XI has a typical myosin structure, consisting of an N-terminal MD, a neck domain comprising six tandem repeats of IQ motifs, a long coiled-coil domain for dimerization, and a C-terminal GTD that binds cargo. The molecular 
mechanism of myosin XI that generates fast cytoplasmic streaming has been investigated using tobacco $175 \mathrm{kDa}$ myosin XI purified from cultured tobacco BY-2 cells. Tobacco $175 \mathrm{kDa}$ myosin XI moves processively along actin filaments with approximately $35 \mathrm{~nm}$ steps ${ }^{10}$. The processive stepping motion is analogous that of myosin Va which is involved in intracellular transport in mammalian cells and moves on actin filaments in a "hand-over-hand" fashion ${ }^{2}$. Although the structural and motile features of myosin $\mathrm{XI}$ are similar to those of myosin Va, the velocity of myosin XI is approximately $5-15 \mathrm{~mm} \mathrm{~s}^{-1}, 10-30$ fold faster than that of myosin Va and the ATPase activity of myosin XI was $76 \mathrm{~s}^{-1}$, six-fold higher than that of myosin Va by high ATPase activity in the MD ${ }^{10} 11$.

Genome analysis revealed that Arabidopsis thaliana possesses 13 myosin XI members ${ }^{12}$. Gene knockout studies have revealed that myosin $\mathrm{XI}-1, \mathrm{XI}-2, \mathrm{XI}-\mathrm{B}, \mathrm{XI}-\mathrm{I}$, and $\mathrm{XI}-\mathrm{K}$ are responsible for the movement of organelles such as the endoplasmic reticulum, Golgi stacks, peroxisomes, and mitochondria ${ }^{13-17}$ concomitant with growth defects ${ }^{15,16,18}$. The full-length cDNAs and promoter regions for all 13 Arabidopsis myosin XI have cloned, and their tissue-specific expression and motile and enzymatic activities identified. The velocities and ATPase activities of the 13 At myosin XIs are significantly different, and the myosins are classified broadly into three groups-high, medium, and low-based on velocity. The velocity grouping appears to be roughly correlated with the tissue-specific expression patterns ${ }^{11}$. Gene knockout analysis indicated that MYA2 and XI-K are the major motor proteins that provide the motive force for cytoplasmic streaming ${ }^{16,17}$. However, among 13 Arabidopsis myosin XIs, the phylogenetical similarity of MYA2 is the closest to tobacco $175 \mathrm{kDa}$ myosin $\mathrm{XI}$. It is unclear whether the motile mechanisms of MYA2 is the same as that of tobacco $175 \mathrm{kDa}$ myosin XI. In this study, the in vitro enzymatic properties and single-molecule motility of recombinant Arabidopsis MYA2 were analyzed. We found that MYA2 has different motile and regulatory properties than those of tobacco $175 \mathrm{kDa}$ myosin XI.

\section{Results}

\section{Constructs}

A schematic diagram of full-length MYA2 (Full) deduced from its amino acid sequence is shown in Fig. 1a. In general, the domain presence and composition were consistent across all 13 At myosin XIs. At myosin XI is composed an MD, a neck domain with six IQ calmodulin-binding motifs to which calmodulin or calmodulin-like proteins bind, a coiled-coil region, and a GTD (Fig. 1a) ${ }^{11,19,20}$. In this study, we generated five types of recombinant MYA2 constructs: full-length (Full) (Fig. 1a); heavy meromyosin which lacked GTD (HMM); motor domain with six IQ motifs (6IQ); motor domain (MD); and globular tail domain (GTD) (Fig. 1b) ${ }^{11}$. These constructs were expressed using a baculovirus transfer system in High Five insect cells. The recombinant MYA2 proteins were purified using both nickel-affinity and Flag-affinity resin, as previously reported ${ }^{11,21}$. 


\section{Inhibitory effects of GTD on actin-activated ATPase activities}

Fig. 2a shows the actin-activated ATPase activities of MYA2 constructs at various actin concentrations. Plots of ATPase activity against actin concentration were found to fit a Michaelis-Menten type curve. Using this curve, we determined the maximum rate of ATP turnover $\left(\mathrm{V}_{\max }\right)$ and the actin concentration at which the ATPase rate reached half of its maximum $\left(K_{m}\right)$. The $V_{\max }$ values of $H M M, 6 I Q$, and MD were 99 $\mathrm{s}^{-1}, 127 \mathrm{~s}^{-1}$, and $56 \mathrm{~s}^{-111}$, respectively (Fig. 2a). In contrast, the $V_{\max }$ value of Full was $8.1 \mathrm{~s}^{-1}$ much smaller than those of HMM, 6IQ, and MD (Fig. 2a). The reason for the low $\mathrm{V}_{\text {max }}$ value of Full was probably due to the inhibitory effect of the GTD on actin-activated ATPase activity. To confirm inhibition by the GTD, the actin-activated ATPase activities of HMM, 6IQ, and MD were measured, adding separately expressed GTD. The GTD inhibited the actin-activated ATPase activities of HMM and 6IQ in a concentration-dependent manner, but not that of MD.

The actin-activated ATPase activities of HMM and 6IQ in the presence of various concentrations of GTD were fit to a hyperbola, defining the affinity of the GTD for HMM and 6IQ as $0.5 \mu \mathrm{M}$ and $0.8 \mu \mathrm{M}$, respectively (Fig. 2b). These results suggest that the GTD inhibits the actin-activated ATPase activities of MYA2, and that this inhibition needs IQ motifs. Fig. 2c shows the actin-activated ATPase activities of 6IQ in the presence of $1 \mu \mathrm{M}$ GTD and the absence of GTD. The $V_{\max }$ values of 6IQ in the absence and the presence of GTD were $127 \mathrm{~s}^{-1}$ and $18 \mathrm{~s}^{-1}$, respectively. The $\mathrm{K}_{\mathrm{m}}$ values of 6IQ for actin in the presence of 1 $\mu \mathrm{M}$ GTD and in the absence of GTD were $106 \mu \mathrm{M}$ and $19 \mu \mathrm{M}$, respectively. Thus, the $\mathrm{V}_{\max }$ value decreased by about seven times, and the affinity for actin $\left(1 / \mathrm{K}_{\mathrm{m}}\right)$ decreased by about six times in the presence of $1 \mu \mathrm{M}$ GTD.

\section{Inhibitory effects of GTD on motility}

6IQ moved actin filaments at $5.2 \mu \mathrm{m} \mathrm{s}^{-1}$, which was similar to the value observed by Full ${ }^{11}$. In the presence of the GTD, the velocity dropped to less than half of the speed without the GDP (Fig. 3a). For most myosins including myosin $\mathrm{XI}$, the actin sliding velocity is mainly determined by the rate of ADP dissociation from acto-myosin $22-25$. The ADP dissociation rate from acto-MD in the presence or absence of the GTD was determined by measuring the decrease in the fluorescence intensity of mant-ADP $22-27$. In the presence of $1 \mu \mathrm{M} \mathrm{GTD}$, the rate decreased to about $1 / 20$ (Fig. 3b). These results suggest that free GTD not bound to cargo inhibits the dissociation of ADP from MYA2-MD (Fig. 3c).

\section{Processivity and stepping dynamics of MYA2}

Next, we investigated the processivity of single MYA2 Full using total internal reflection fluorescence microscopy (TIRFM). Although MYA2 fused with GFP at the MD showed non-processive motion along an 
actin filament at a saturating $1 \mathrm{mM}$ ATP, it processively moved in one direction at concentration of $1 \mathrm{mM}$ ATP. The fluorescence of GFP showing processive motions photobleached in a single- or double-step, strongly suggesting single-molecule processivity (Figure S1).

Then, to dissect the stepping dynamics during the processive motion, we attached a fluorescent quantum $\operatorname{dot}(\mathrm{QD})$ to an MD, using a Halo-tag domain at the N-terminus, in an MYA2 dimer, and tracked the motion with $2 \mathrm{~nm}$ precision and $33 \mathrm{~ms}$ time resolution using the FIONA method ${ }^{28}$. Stepwise motion could be detected, and the step size distribution was fit to a three Gaussian function with peaks at $60 \mathrm{~nm}$ (large forward steps), $29 \mathrm{~nm}$ (short forward steps) and -28 nm (backward steps) (Fig. 4a and b). To exclude the possibility that steric hindrance by the QD was responsible for the stepping dynamics, we also examined tetramethyl rhodamine (TMR)-labeled MYA2. The velocity and run length of QD-labeled MYA2 were similar to those for TMR labeled MYA2 (Table S1), and the three types of steps were also resolved for TMR labeled MYA2 (Figure S2). These results indicated that steric hindrance due to QD labeling at the MD was not significant. A histogram of the dwell time just before the forward steps was fit to a convolution of two exponentials (tk $\left.{ }^{2} \exp [-k t]\right)$, in which $k$ is a rate constant (Figure S3). This is reasonable if we assume that the large $(60 \mathrm{~nm})$ and short $(29 \mathrm{~nm})$ forward steps are caused by a hand-over-hand motion and inchworm-like motion, respectively ${ }^{29}$.

\section{Weak force generation of MYA2}

To examine the force generation of MYA2, we attached a fluorescent polystyrene bead to the C-terminal tail and applied force using an optical trap. Fig. 4c shows the typical trace of a single MYA2 full under load at $1 \mu \mathrm{M}$ ATP, and the step size histogram is shown in Fig. $4 \mathrm{~d}$. The histogram was fitted by two Gaussian functions with peaks at $31 \mathrm{~nm}$ (forward steps), and $-31 \mathrm{~nm}$ (backward steps). Fig. 4e shows the histogram of the stall force; the mean stall force was $0.86 \mathrm{pN}$.

\section{Discussion}

In this study, we analyzed the in vitro enzymatic properties of MYA2 which is known to be a major driving force of cytoplasmic streaming. The $V_{\max }$ value of the actin-activated ATPase activities measured using recombinant HMM and 6IQ, and the MD of MYA2 were higher than that of myosin $\mathrm{V}$, and similar to that of tobacco $175 \mathrm{kDa}$ myosin XI (Fig. 2A). This high ATPase activity is consistent with MYA2 acting as a driving force for cytoplasmic streaming. In contrast, the $V_{\max }$ value of the ATPase activity of full length MYA2 was much lower than those of $\mathrm{HMM}, 6 \mathrm{IQ}$, and MD. This result indicated that the activity of MYA2 was inhibited by the GTD. Exogenous addition of the GTD inhibited the actin-activated ATPase activities of HMM and 6IQ in a concentration-dependent manner, but not that of MD, suggesting that GTD inhibits ATPase activity via the IQ motif. Such GTD suppression was not observed in full-length tobacco $175 \mathrm{kDa}$ myosin XI purified from tobacco BY-2 cells ${ }^{10}$. Autoinhibition using GTD has been reported in myosin $\mathrm{V}$, myosin VIIA, and myosin X. This intra-molecular regulation could avoid unproductive interactions with actin filaments, and is essential for the recycling of free myosins after unloading cargo. The same intra- 
molecular regulation in MYA2 suggests that MYA2 is not merely responsible for driving continuous cytoplasmic streaming, but also for more complex transport equipped with a recycling system. It has recently been reported that MYA2 is involved in several intracellular transports and functions, such as processing-body movement, pathogen responses, auxin responses, cell death, cell division, regulation of exocytosis, lipid body transport $18,30-32$.

The duty ratio of myosin is defined as the ratio of time spent strongly bound to actin to the cycle time of actin-activated ATPase. The time spent strongly bound to actin is the sum of thr acto-myosin-ADP (AM.ADP) and acto-myosin (AM) states ${ }^{33}$. Because the AM state is very short for most myosins, the time spent strongly bound to actin is almost equal to that spent in the AM.ADP state, which is approximated by the reciprocal of the ADP dissociation rate from acto-6IQ $\left(1 / 403 \mathrm{~s}^{-1}=2.48 \mathrm{~ms}\right)$. The cycle time of actin-activated ATPase is approximated by the reciprocal of the Vmax value of $6 I \mathrm{Q}$ is $32 \%(100 \times 2.48$ $\mathrm{ms} / 7.87 \mathrm{~ms}$ ), showing that MYA2 is a non-processive myosin.

The single-molecule motility of MYA2 revealed its unique motility, which is quite different from that previously reported for tobacco $175 \mathrm{kDa}$ myosin $\mathrm{XI}^{10}$. Optical nanometry of single MYA2 molecules using TIRF microscopy revealed that MYA2 was non-processive at high ATP concentrations, as suggested by ATPase activities. However, MYA2 demonstrated processive motion on an actin filament at low ATP concentrations. FIONA revealed that MYA2 moved with three different step sizes: $-28 \mathrm{~nm}, 29 \mathrm{~nm}$, and 60 $\mathrm{nm}$. This result indicated that MYA2 uses two different stepping modes, hand-over-hand and inchwormlike (Fig. 5). Force measurement using optical trapping showed that the stall force of MYA2 was $0.85 \mathrm{pN}$, which was similar to that of tobacco $175 \mathrm{kDa}$ myosin $\mathrm{XI}(0.5 \mathrm{pN})$ and less than half that of myosin $\mathrm{V}$ (2$3 \mathrm{pN})^{34,35}$. These results indicated that MYA2 is a more flexible motor than myosin $\mathrm{V}$, which is responsible for vesicle transport in animal cell. This flexibility is thought to avoid interference between myosin molecules when many motors work closely together. If a certain myosin interferes with the myosin in front of it, it is possible to briefly pause the movement of trailing myosin by it stepping back a half step, without backstepping. MYA2 may be suitable motor for driving large organelle such as the ER with multiple molecules simultaneously. Tobacco $175 \mathrm{kDa}$ myosin XI was phylogenetically the closest MYA2 among the 13 Arabidopsis myosin XI members. However, considerable differences were found in the mode of movement and in the regulation, suggesting that myosin Xls have diverse molecular functions.

\section{Methods}

\section{Protein engineering, and expression}

A baculovirus transfer vector for pFastBacMYA2 was generated as follows. MYA2 (AT5g43900) cDNA cloned from Arabidopsis seedlings was mutated to create an $\mathrm{Ncol}$ site at the upstream region of the nucleotide sequence encoding residue 1, and an Agel site in the downstream region of the nucleotide sequence encoding residue 4515 of MYA2. These sequences were cut with $\mathrm{Ncol}$ and Agel and the 
fragment was ligated with the Ncol-Agel fragment of the pFastBac MD with FLAG-tag ${ }^{25}$. The resulting construct, MYA2, encodes the N-terminal tag (MSYYHHHHHHDYKDDDDKNIPTTENLYFQGA) containing the sequence of a (His)6-tag and a FLAG-tag (DYKDDDDK), residues 1-1505 of MYA2, a flexible linker (GGG), a Myc-epitope sequence (EQKLISEEDL), and a (His)8-tag. A baculovirus transfer vector for pFastBac Halo-tag fused MYA2 was generated as follows. The pFN21A (HaloTag (R) 7) CMV Flexi(R) Vector (Promega Corporation, Madison, USA) was mutated to create an Ncol site downstream of the nucleotide sequence encoding the HaloTag 7 open reading frame, and was cut with Ncol. The Ncol digestion fragment was ligated with the Ncol digestion fragment of pFastBacMYA2. The resultant construct, Halo-tag fused MYA2, encodes the N-terminal tag

(MSYYHHHHHHDYKDDDDKNIPTTENLYFQGA) containing the sequence of a (His)6-tag, a FLAG-tag (DYKDDDDK), and a Halo-tag 7, residues 1-1505 of MYA2, a flexible linker (GGG), a Myc-epitope sequence (EQKLISEEDL), and a (His)8-tag. A baculovirus transfer vector for pFastBac Arabidopsis calmodulin was generated as follows. Arabidopsis calmodulin (AT3G56800.1) cDNA cloned from Arabidopsis seedling was mutated to create an Xbal site upstream of the nucleotide sequence encoding residue 1 , and an Xhol site downstream of the nucleotide sequence encoding residue 448 of Arabidopsis calmodulin. The Xbal-Xhol digestion fragment was ligated with the Xbal-Xhol digestion fragment of pFastBac 1 (Invitrogen, Carlsbad, CA, USA). To express MYA2 and Halo-tag fused MYA2, $800 \mathrm{ml}$ of a culture of High Five TM cells (Invitrogen) were infected with viruses expressing the respective constructs. Arabidopsis calmodulin was co-produced by co-infection with virus expressing Arabidopsis calmodulin. The infected cells were cultured in Erlenmeyer flasks $(1 \mathrm{~L} \mathrm{x} \mathrm{4})$ at $28^{\circ} \mathrm{C}$ and, shaken at $130 \mathrm{rpm}$, for $43 \mathrm{~h}$. The study complies with national guidelines in Japan.

\section{Protein purification}

Protein purification was performed as previously described with some modification ${ }^{33}$. Cells were harvested and washed with $150 \mathrm{mM} \mathrm{NaCl}, 1 \mathrm{mM}$ EGTA, and $10 \mathrm{mM}$ Hepes, $\mathrm{pH}$ 7.4. The pelleted cells were suspended with $2 \mathrm{vol} / \mathrm{g}$ cells of buffer A (30 mM Hepes, pH 7.4, $200 \mathrm{mM} \mathrm{KCl,} 5 \mathrm{mM} \mathrm{MgCl}$, $4 \mathrm{mM} \mathrm{ATP,} 1$ $\mathrm{mM}$ EGTA, $1 \mathrm{mM}$ DTT, and a mixture of protease inhibitors). Then $2 \mathrm{vol} / \mathrm{g}$ cells of buffer $\mathrm{A}$ containing $2 \%$ Nonidet P-40 was added and mixed. After incubation on ice for $10 \mathrm{~min}$, the lysate was centrifuged at $228,000 \times \mathrm{g}$ for $30 \mathrm{~min}$. The supernatant was mixed with $0.3 \mathrm{ml}$ of anti-FLAG M2 affinity resin (SigmaAldrich, St. Louis, MO, USA) in a $50 \mathrm{ml}$ tube on a rotating wheel for $1 \mathrm{~h}$ at $4^{\circ} \mathrm{C}$. The resin suspension was then loaded on a column and washed with $30 \mathrm{ml}$ of buffer A containing $1 \mu \mathrm{M}$ mouse calmodulin. Halotag fused MYA2 was biotinylated by incubation with buffer A containing $10 \mu \mathrm{M}$ of HaloTag PEG-Biotin Ligand (Promega) in the column for 10 min. MYA2 and Halo-tag fused MYA2 was eluted with buffer A containing $0.2 \mathrm{mg} / \mathrm{ml}$ of $3 x F L A G$ peptide (Sigma-Aldrich). Arabidopsis calmodulin was expressed in High Five TM cells by infecting them with virus expressing Arabidopsis calmodulin, and purified using the method of Awata et al. ${ }^{36}$.

\section{ATPase activity}


ATPase activities were determined by measuring released phosphate as previously described ${ }^{37}$. The reaction mixtures for the assay of actin-activated $\mathrm{Mg}^{2+}$-ATPase activity contained were done in $25 \mathrm{mM}$ $\mathrm{KCl}, 4 \mathrm{mM} \mathrm{MgCl}, 25 \mathrm{mM}$ Hepes-KOH (pH 7.4), $2 \mathrm{mM} \mathrm{ATP}, 1 \mathrm{mM} \mathrm{DTT}$, and $1 \mathrm{mg} / \mathrm{ml} \mathrm{BSA}$ and at 25 ${ }^{\circ} \mathrm{C}$, 0.125 - $4 \mathrm{mg} / \mathrm{ml}$ F-actin.

\section{in vitro gliding assay}

The velocity was measured using an anti-myc antibody-based version of the in vitro actin filament gliding assay as previously described ${ }^{25}$. The velocity of actin filaments was measured in $150 \mathrm{mM} \mathrm{KCl}, 4 \mathrm{mM}$ $\mathrm{MgCl}_{2}, 25 \mathrm{mM}$ Hepes-KOH (pH 7.4), 2 mM ATP, 10 mM DTT and oxygen scavenger system $(120 \mu \mathrm{g} / \mathrm{ml}$ glucose oxidase, $12.8 \mathrm{mM}$ glucose, and $20 \mu \mathrm{g} / \mathrm{ml}$ catalase) at $25^{\circ} \mathrm{C}$. Average sliding velocities determined by measuring the displacements of actin filaments.

\section{Single-molecule imaging}

Single-molecule imaging was performed as previously described ${ }^{29}$. Briefly, Q-dot585 streptavidin conjugations (Invitrogen) and biotinylated MYA2 were mixed at a ratio of 1:1 and incubated prior to use overnight on ice. Assay buffer (AB; $30 \mathrm{mM} \mathrm{HEPES-KOH} \mathrm{[pH} \mathrm{7.8],} 25 \mathrm{mM} \mathrm{KCl}, 5 \mathrm{mM} \mathrm{MgCl}$, and $2 \mathrm{mM}$ EGTA) was prepared before each experiment. Sample chambers were assembled using double-sided transparent tape (Scotch) and dried coverslips. Actin filaments were quickly flowed into sample chambers in which a-actinin molecules were directly adhered to a glass slide. The glass surface was then coated with $5 \mathrm{mg} / \mathrm{ml}$ casein. A 1: I Q-dot-MYA2 mixture was diluted 100 times in motility buffer (MB; AB plus, $1 \mathrm{mM}$ ATP, $0.2 \mathrm{mg} / \mathrm{mL}$ glucose oxidase, $4.5 \mathrm{mg} / \mathrm{mL}$ glucose, $36 \mathrm{mg} / \mathrm{mL}$ catalase, $1 \% \mathrm{v} / \mathrm{v} 2$ -

mercaptoethanol ${ }^{38}, 2 \mathrm{mM}$ phosphocreatine, $0.1 \mathrm{mg} / \mathrm{mL}$ creatine phosphokinase ${ }^{39}, 0.1 \mathrm{mg} / \mathrm{ml}$ calmodulin). This mixture was added into the sample chamber, and the chamber was sealed with nail polish and observed immediately. Q-dot conjugated MYA2 movement was imaged using TIRFM, and the excitation was provided by a $405 \mathrm{~nm}$ laser light (Compass405-50CW; Coherent, Santa Clara, CA, USA). The fluorescent photons were collected with a back-illuminated EMCCD camera (DV887ECS-BV; Andor Technology, Belfast, Northern Ireland). The fluorescence was passed through a dichroic mirror (DML557 nm; Asahi Spectra, Tokyo, Japan) and emission filter (FF01-593/40-25, Semrock, Rochester, NY, USA). Image acquisition was performed by commercial software (Andor, SOLIS software). The sample was kept at ambient temperature during data collection $\left(27^{\circ} \mathrm{C} \pm 2^{\circ} \mathrm{C}\right)$. Exported eight-bit data was analyzed by a program custom written in LabVIEW (National Instruments, Austin, TX, USA). The spot center for each frame was determined using a two-dimensional Gaussian fit according to a published method ${ }^{40} 28$. The accuracy of detection of the spot center was $2.0 \mathrm{~nm}$. All steps were analyzed by an automated stepfinding algorithm $\mathrm{m}^{4}$.

\section{Optical trapping}


Optical trapping was performed as previously described, with some modifications ${ }^{42}$. Briefly, carboxylate modified polystyrene beads (Invitrogen; $0.2 \mathrm{~mm}$ in diameter) were cross-linked to c-Myc monoclonal antibody (Clontech, Shiga, Japan) and coated with $10 \mathrm{mg} / \mathrm{ml} \mathrm{BSA}$. One microliter of the beads and c-myc tagged MYA2 were mixed and incubated for $30 \mathrm{~min}$ in $A B$ containing $10 \mathrm{mg} / \mathrm{ml} \mathrm{BSA}$ at a ratio of 1 to 20 . The ratio was adjusted so that less than $10 \%$ of the beads displayed movement. Sample chambers were assembled using double-sided transparent tape (Scotch) and dried coverslips. Fluorescent dye-labeled actin filaments were quickly flowed into sample chambers in which a-actinin molecules were directly adhered to a glass slide. The glass surface was then coated with $5 \mathrm{mg} / \mathrm{ml}$ casein. One microliter of MYA2 coated beads was diluted 100 times in MB. This mixture was added to the sample chamber, and the chamber was sealed with nail polish and observed immediately. The optics of the optical trapping and detection of the bead positions were done as previously described ${ }^{39,42}$. Bead displacements were recorded at a sampling rate of $24 \mathrm{kHz}$ with a bandwidth of $10 \mathrm{kHz}$. The load exerted on the beads was calculated from the bead displacement multiplied by the trap stiffness $(\sim 7.4 \mathrm{fN} / \mathrm{nm})$, which was determined from the variance of the Brownian motion of a trapped bead by the equipartition theorem of energy. We determined the step sizes after passing the data through a low pass filter with a bandwidth of $200 \mathrm{~Hz}$, using a custom program in DADiSP (CAE Solutions, Burlington, MA, USA) and Visual C++ (Microsoft, Redmond, WA, USA). All steps were checked by eye. To determine the precise step size at low loads, we calculated the elastic component in our single trapping geometry, and estimated the correction factor depending on $\operatorname{load}^{43}$.

\section{Declarations}

\section{Acknowledgements}

This work was supported by JSPS KAKENHI (no. 920001009 , no. 23770060 and no. 25221103 to M.T.) (no. JP 20K06583, no. JP 17K07436, 15H01309 to K.I.) (no. 21710120 and no. 26440131 to M.I.), and from the Japan Science and Technology Agency, ALCA, JPMJAL1401 (to M.T. and K.I.).

\section{Author contributions statement}

M.T., K.I, and M.I. conceived the idea and design the study. T.H., T.M., and N.S. conducted the experiments, T.H., K.I., and M.I. analyzed the results. M.T., K.I, and M.I. contributed to the writing of the manuscript. All authors reviewed the manuscript.

\section{Competing interests}

The authors declare no competing interests.

\section{References}


1. Kollmar, M. \& Mühlhausen, S. Myosin repertoire expansion coincides with eukaryotic diversification in the Mesoproterozoic era. BMC Evol. Biol, 17, 211-212 (2017).

2. Sweeney, H. L. \& Holzbaur, E. L. F. Motor Proteins. Cold Spring Harb Perspect. Biol, 10, a021931 https://doi.org/10.1101/cshperspect.a021931 (2018).

3. Titus, M. A. Myosin-Driven Intracellular Transport. Cold Spring Harb Perspect. Biol, 10, https://doi.org/a021972 (2018).

4. Hartman, M. A., Finan, D., Sivaramakrishnan, S. \& Spudich, J. A. Principles of unconventional myosin function and targeting. Annu. Rev. Cell Dev. Biol, 27, 133-155 (2011).

5. Heissler, S. M. \& Sellers, J. R. Kinetic Adaptations of Myosins for Their Diverse Cellular Functions. Traffic, 17, 839-859 (2016).

6. Liu, J., Taylor, D. W., Krementsova, E. B., Trybus, K. M. \& Taylor, K. A. Three-dimensional structure of the myosin V inhibited state by cryoelectron tomography. Nature, 442, 208-211 (2006).

7. Li, J. F. \& Nebenführ, A. The tail that wags the dog: the globular tail domain defines the function of myosin V/XI. Traffic, 9, 290-298 (2008).

8. Nascimento, A. F. Z. et al. Structural insights into functional overlapping and differentiation among myosin V motors. J. Biol. Chem, 288, 34131-34145 (2013).

9. Shimmen, T. The sliding theory of cytoplasmic streaming: fifty years of progress. J. Plant Res, 120, 31-43 (2007).

10. Tominaga, M. et al. Higher plant myosin XI moves processively on actin with $35 \mathrm{~nm}$ steps at high velocity. EMBO J, 22, 1263-1272 (2003).

11. Haraguchi, T. et al. Functional Diversity of Class XI Myosins in Arabidopsis thaliana. Plant Cell Physiol, 59, 2268-2277 (2018).

12. Reddy, A. S. \& Day, I. S. Analysis of the myosins encoded in the recently completed Arabidopsis thaliana genome sequence. Genome Biol. 2, RESEARCH0024-research0024. Epub $2001 \mathrm{Jul}$ 3(2001).

13. Peremyslov, V. V., Klocko, A. L., Fowler, J. E. \& Dolja, V. V. Arabidopsis Myosin XI-K Localizes to the Motile Endomembrane Vesicles Associated with F-actin. Front. Plant. Sci, 3, 184 (2012).

14. Peremyslov, V. V., Prokhnevsky, A. I., Avisar, D. \& Dolja, V. V. Two class XI myosins function in organelle trafficking and root hair development in Arabidopsis. Plant Physiol, 146, 1109-1116 (2008).

15. Peremyslov, V. V., Prokhnevsky, A. I. \& Dolja, V. V. Class XI myosins are required for development, cell expansion, and F-Actin organization in Arabidopsis. Plant Cell, 22, 1883-1897 (2010).

16. Prokhnevsky, A. I., Peremyslov, V. V. \& Dolja, V. V. Overlapping functions of the four class XI myosins in Arabidopsis growth, root hair elongation, and organelle motility. Proc. Natl. Acad. Sci. U. S. A. 105, 19744-19749(2008).

17. Ueda, H. et al. Myosin-dependent endoplasmic reticulum motility and F-actin organization in plant cells. Proc. Natl. Acad. Sci. U. S. A. 107, 6894-6899(2010). 
18. Ojangu, E. et al. Myosins XI-K, XI-1, and XI-2 are required for development of pavement cells, trichomes, and stigmatic papillae in Arabidopsis. BMC plant biology, 12, 81 (2012).

19. Tominaga, M. \& Nakano, A. Plant-Specific Myosin XI, a Molecular Perspective. Frontiers in plant science, 3, 211 (2012).

20. Tominaga, M. \& Ito, K. The molecular mechanism and physiological role of cytoplasmic streaming. Curr. Opin. Plant Biol, 27, 104-110 (2015).

21. Haraguchi, T., Tominaga, M., Nakano, A., Yamamoto, K. \& Ito, K. Myosin XI-I is Mechanically and Enzymatically Unique Among Class-XI Myosins in Arabidopsis. Plant Cell Physiol, 57, 1732-1743 (2016).

22. De La Cruz, E. M., Wells, A. L., Rosenfeld, S. S., Ostap, E. M. \& Sweeney, H. L. The kinetic mechanism of myosin V. Proc. Natl. Acad. Sci. U. S. A. 96, 13726-13731(1999).

23. De La Cruz, E. M., Ostap, E. M. \& Sweeney, H. L. Kinetic mechanism and regulation of myosin VI. J. Biol. Chem, 276, 32373-32381 (2001).

24. Trybus, K. M., Krementsova, E. \& Freyzon, Y. Kinetic characterization of a monomeric unconventional myosin V construct. J. Biol. Chem, 274, 27448-27456 (1999).

25. Ito, K. et al. Kinetic mechanism of the fastest motor protein, Chara myosin. J. Biol. Chem, 282, 19534-19545 (2007).

26. Kambara, T., Komaba, S. \& Ikebe, M. Human myosin III is a motor having an extremely high affinity for actin. J. Biol. Chem, 281, 37291-37301 (2006).

27. Haraguchi, T. et al. Molecular characterization and subcellular localization of Arabidopsis class VIII myosin, ATM1. J. Biol. Chem, 289, 12343-12355 (2014).

28. Yildiz, A. et al. Myosin V walks hand-over-hand: single fluorophore imaging with 1.5-nm localization., 300, 2061-2065 (2003).

29. Nishikawa, S. et al. Switch between large hand-over-hand and small inchworm-like steps in myosin VI., 142, 879-888 (2010).

30. Ojangu, E. L. et al. Class XI Myosins Contribute to Auxin Response and Senescence-Induced Cell Death in Arabidopsis. Front. Plant. Sci, 9, 1570 (2018).

31. Abu-Abied, M. et al. Myosin XI-K is involved in root organogenesis, polar auxin transport, and cell division. J. Exp. Bot, 69, 2869-2881 (2018).

32. Zhang, W., Huang, L., Zhang, C. \& Staiger, C. J. Arabidopsis myosin XIK interacts with the exocyst complex to facilitate vesicle tethering during exocytosis.Plant Cel/(2021).

33. Ito, K., Yamaguchi, Y., Yanase, K., Ichikawa, Y. \& Yamamoto, K. Unique charge distribution in surface loops confers high velocity on the fast motor protein Chara myosin. Proc. Natl. Acad. Sci. U. S. A. 106, 21585-21590(2009).

34. Mehta, A. D. et al. Myosin-V is a processive actin-based motor. Nature, 400, 590-593 (1999).

35. Iwaki, M., Iwane, A. H., Ikezaki, K. \& Yanagida, T. Local heat activation of single myosins based on optical trapping of gold nanoparticles. Nano Lett, 15, 2456-2461 (2015). 
36. Awata, J., Saitoh, K., Shimada, K., Kashiyama, T. \& Yamamoto, K. Effects of Ca2 + and Calmodulin on the Motile Activity of Characean Myosin In vitro. Plant Cell Physiol, 42, 828-834 (2001).

37. Ito, K. et al. Recombinant motor domain constructs of Chara corallina myosin display fast motility and high ATPase activity. Biochem. Biophys. Res. Commun, 312, 958-964 (2003).

38. Harada, Y., Sakurada, K., Aoki, T., Thomas, D. D. \& Yanagida, T. Mechanochemical coupling in actomyosin energy transduction studied by in vitro movement assay. J. Mol. Biol, 216, 49-68 (1990).

39. Iwaki, M., Iwane, A. H., Shimokawa, T., Cooke, R. \& Yanagida, T. Brownian search-and-catch mechanism for myosin-VI steps. Nat. Chem. Biol, 5, 403-405 (2009).

40. Thompson, R. E., Larson, D. R. \& Webb, W. W. Precise nanometer localization analysis for individual fluorescent probes. Biophys. J, 82, 2775-2783 (2002).

41. Kerssemakers, J. W. et al. Assembly dynamics of microtubules at molecular resolution. Nature, $\mathbf{4 4 2 ,}$ 709-712 (2006).

42. Iwaki, M. et al. Cargo-binding makes a wild-type single-headed myosin-VI move processively. Biophys. J, 90, 3643-3652 (2006).

43. Kojima, H., Muto, E., Higuchi, H. \& Yanagida, T. Mechanics of single kinesin molecules measured by optical trapping nanometry. Biophys. J, 73, 2012-2022 (1997).

\section{Figures}

a

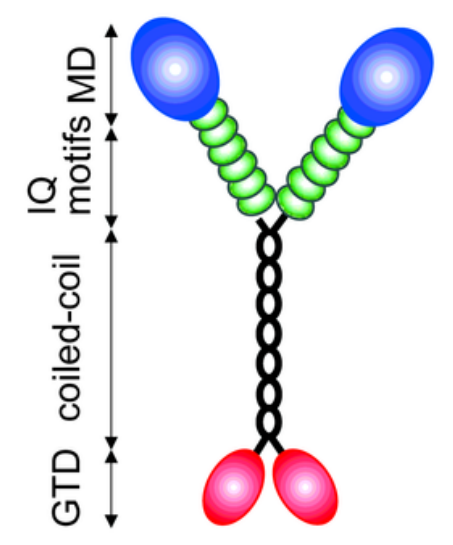

Full b

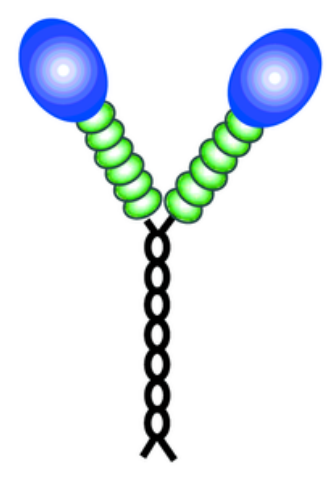

HMM

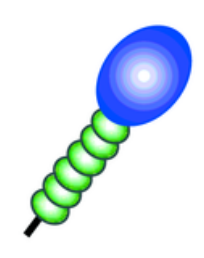

$6 I Q$
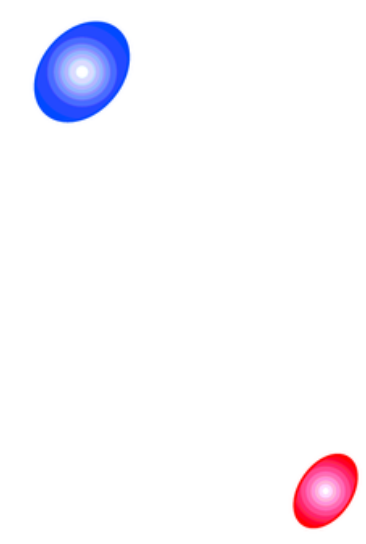

\section{Figure 1}


Schematic diagrams of the MY2 constructs used in this work. (a) Full: Full length (Native) construct comprising MD, six IQ motifs to which six calmodulins bind, coiled-coil, and GTD domains. (b) HMM: HMM construct comprising of MD, six IQ motifs to which six calmodulins bind, and a coiled-coil domain. 6IQ: 6IQ construct comprising of MD and six IQ motifs to which six calmodulins bind. MD: MD construct comprising only MD. GTD: GTD construct comprising only GTD.
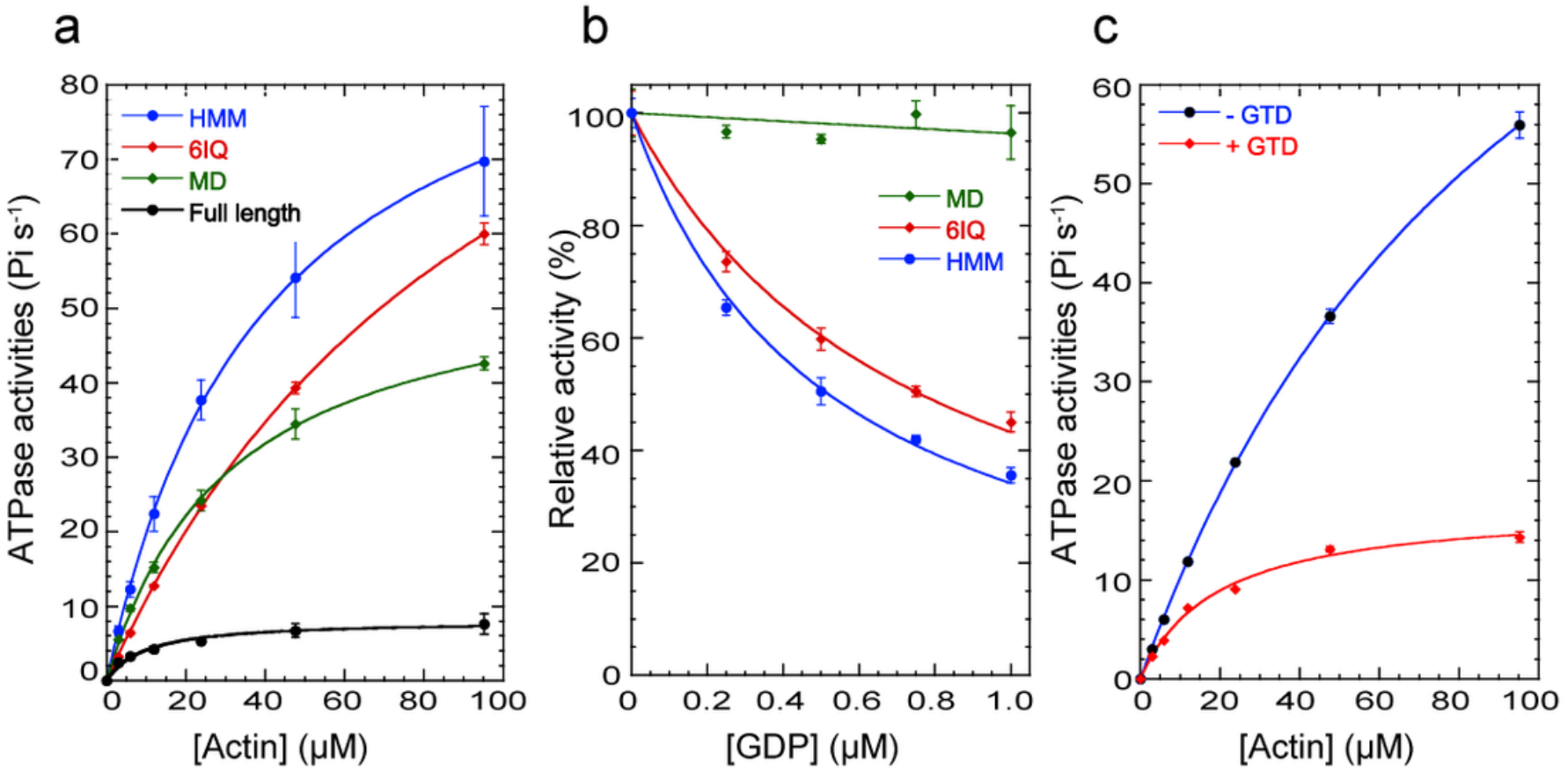

\section{Figure 2}

(a) Actin-activated Mg2+-ATPase activities of Full length, HMM, 6IQ, and MD. Mg2+-ATPase activities in the presence of various actin concentrations. The data were fitted using Michaelis-Menten dynamics, and the Vmax and Kapp values were measured. (b) Inhibitory effects on Mg2+-ATPase activities of HMM, $M D$, and $61 \mathrm{Q}$ of various concentrations of GTD in the presence of $1 \mathrm{mg} / \mathrm{ml}$ actin. (c) Inhibitory effects of Mg2+-ATPase activities of HMM by $1 \mu \mathrm{M}$ GTD in the presence of various actin concentrations. 

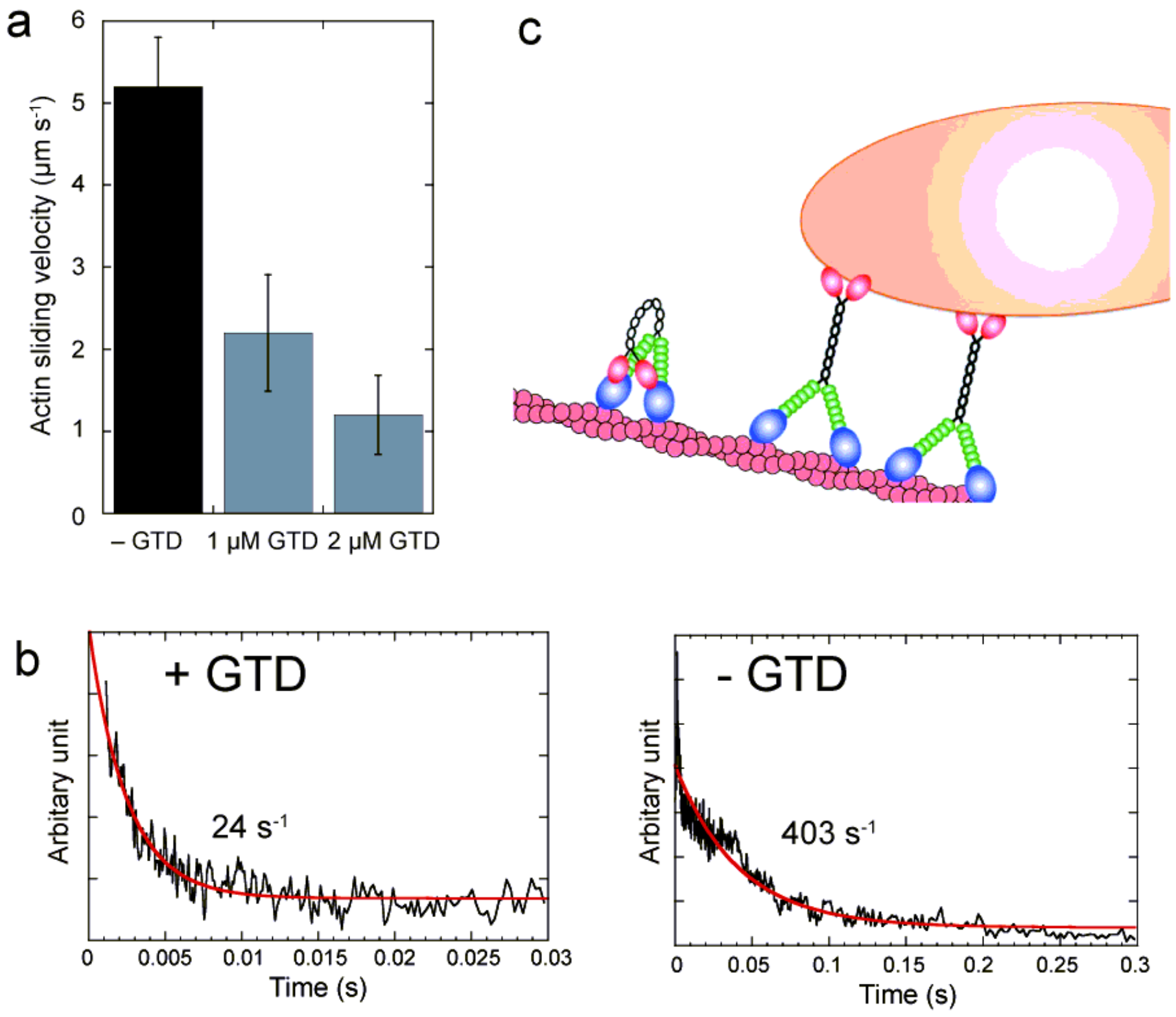

Figure 3

(a) Inhibitory effects of actin sliding velocities of HMM by $1 \mu$ M GTD and $2 \mu M$ GTD. (b) Mant-ADP dissociation from acto-6IQ in the presence of $1 \mu \mathrm{M}$ GTD and absence of GTD. The dissociation rates of mant-ADP from 6IQ were measured using fluorescence energy transfer between the tryptophans of MD of $6 I Q$ and mant-ADP. The transient shown is the average of six separate recordings. The red line is a single exponential fit yielding a rate constant of $24 s^{-1}$ in the presence of $1 \mu \mathrm{M}$ GTD (left) and $430 s^{-1} 1$ in the absence of GTD (right). The average value from three independent assays was $22 \pm 3.4 \mathrm{~s}-1$ in the presence of $1 \mu \mathrm{M}$ GTD and $430 \pm 40 \mathrm{~s}-1$ in the absence of $1 \mu \mathrm{M}$ GTD. (c) A model of the mechanism by which GTD inhibits the movement of MYA2. When GTD is not bound to the organelle, it interacts with MD and inhibits ADP dissociation from MD, thus slowing down the velocity of MYA2, whereas when GTD is bound to the organelle, it does not interact with MD and thus does not inhibit MYA2 movement. 
B
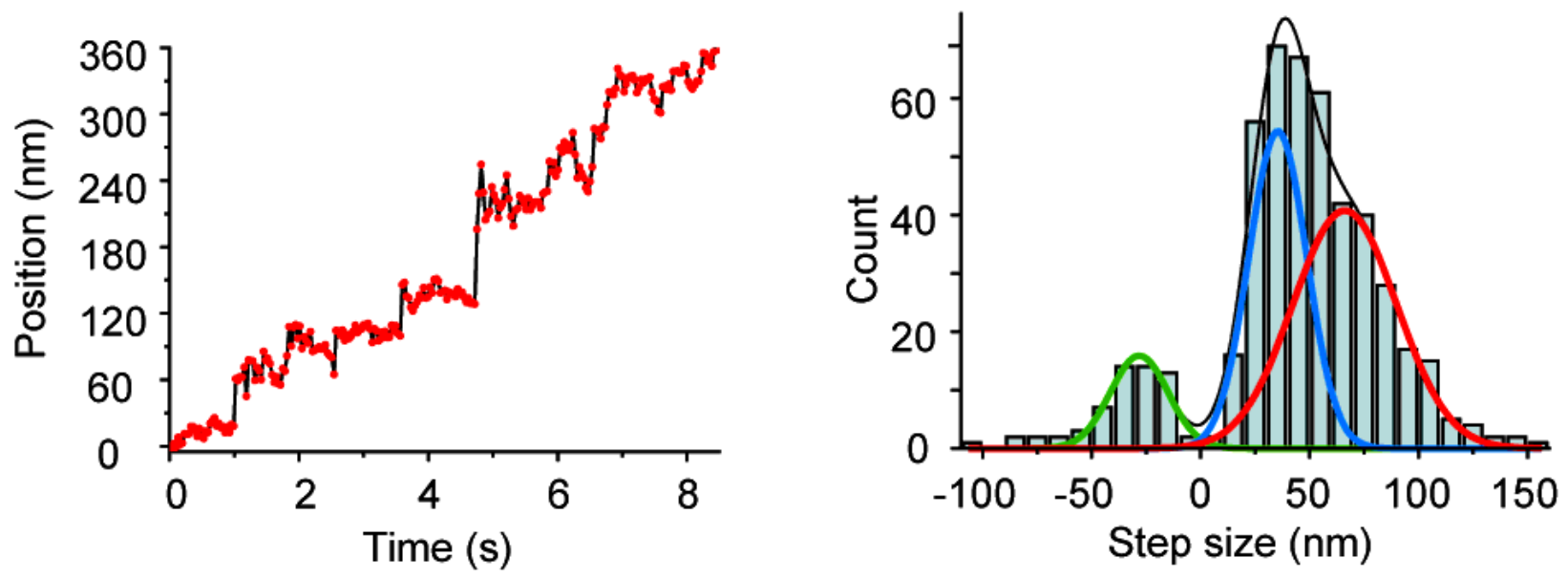

C
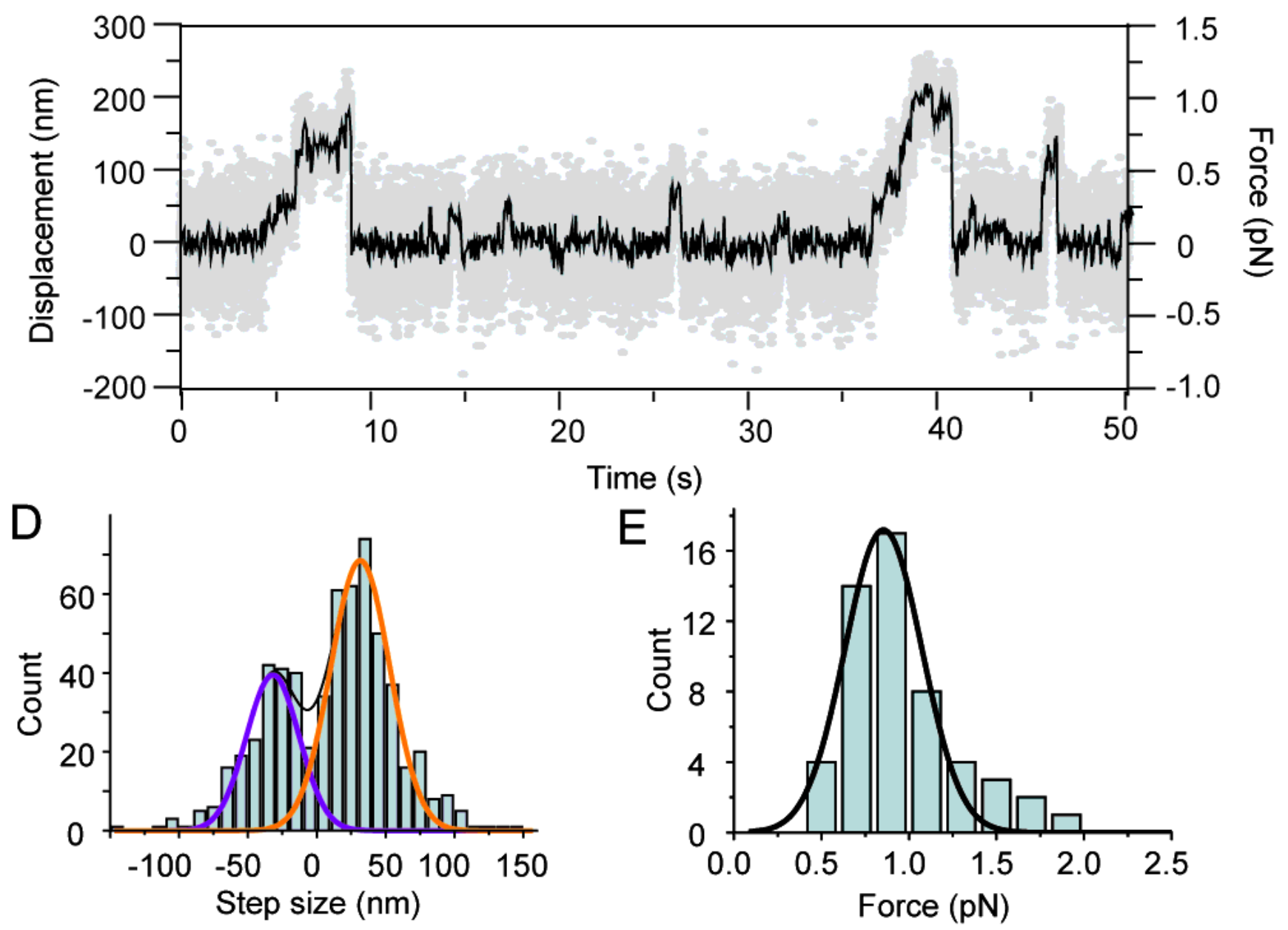

Figure 4

Stepping dynamics and force measurement of MYA2. (a) QD attached to a motor domain in a MYA2 dimer was observed using TIRFM at $2 \mathrm{~nm}$ and $33 \mathrm{~ms}$ spatiotemporal resolution.ATP concentration, 10 $1 \mathrm{M}$. (b) Step size histogram of the motor domain. The histogram fitted to three Gaussian functions with peaks at $60.5 \pm 14.8 \mathrm{~nm}$ (red line), $29.3 \pm 6.9 \mathrm{~nm}$ (blue line), and $-28.1 \pm 9.9 \mathrm{~nm}$ (green line), respectively. Black line indicates the convolution of three Gaussian functions. (c) Typical trace of optical trapping 
nanometry at 10M ATP. Gray line: raw trace; Black line: same data passed through $200 \mathrm{~Hz}$ low pass filter. (d) Step size histogram of an optically trapped bead attached to the MYA2 C-terminal tail. The histogram was fitted to two Gaussian functions with peaks at $31.6 \pm 15.2 \mathrm{~nm}$ (orange line), and $-31.9 \pm 13.6 \mathrm{~nm}$ (purple line), respectively. Black line indicates the convolution of the two Gaussian functions. (e) Histogram of MYA2 stall force. The histogram was fitted to single Gaussian function with peaks at $0.85 \pm$ $0.16 \mathrm{pN}$ (black line).
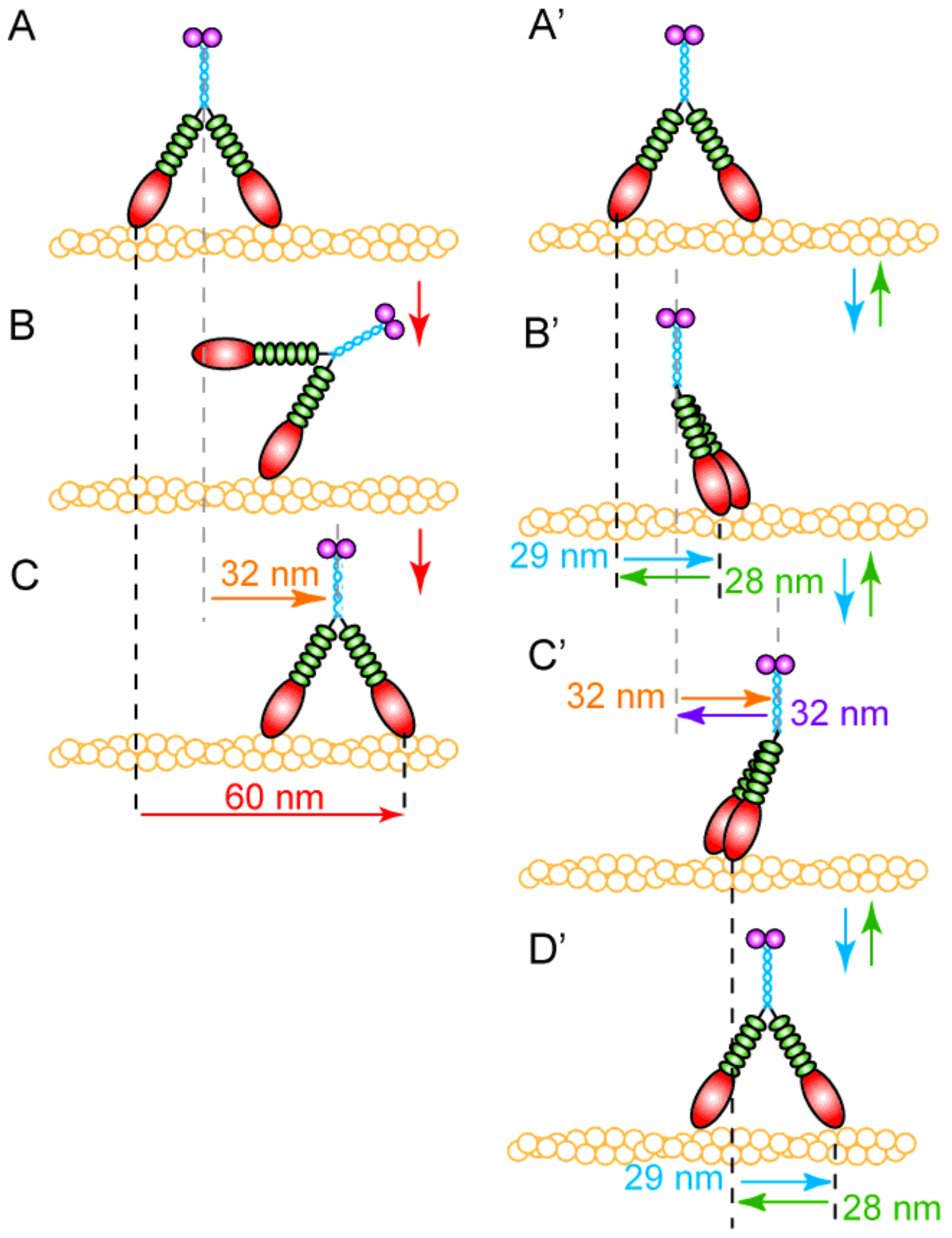

Figure 5 
Walking model of MYA2. Left ( $a, b$ and $c)$, large hand-over-hand steps; right ( $a^{\prime}, b^{\prime}, c^{\prime}$ and d'), small inchworm-like steps. Colors denote the following: N-terminal motor domain (red), lever arm (six calmodulins, green), coiled-coil domain (blue), and the globular tail domain (purple). Red, blue and green arrows indicate the step of the motor domain for large, small, and backward steps, respectively, observed using TIRFM. Orange and purple arrows indicate the step of the tail domain for forward and backward steps, respectively, observed by optical trapping.

\section{Supplementary Files}

This is a list of supplementary files associated with this preprint. Click to download.

- SupplementaryMaterials.pdf 